\section{New Ion Sources for Mass Spectroscopy}

For use in connexion with a new mass-spectrograph, I have recently developed a new type of ion source in which positively charged atoms are formed by sparks between solid electrodes in a high vacuum. Spectroscopic studies in recent years have shown that these vacuum sparks are efficient sources of multiply charged ions. Several forms of sparks were tried; the 'trembleur a vide', the 'hot-sparks' from a large condenser discharge, and finally a spark coupled inductively to a high-frequency oscillating spark circuit. This latter has proved very successful, and an abundance of ions has been obtained, thus far from the following elements: platinum, gold, tungsten, tin, copper, nickel, iron, aluminium, carbon, beryllium and lithium.

The ions were analysed provisionally by the Thomson parabola method after being accelerated by about 20,000 volts. The presence of multiply charged ions is in most cases very striking; spots occur with fractional electrostatic deffections, indicating ions that changed their charge before reaching the electric and magnetic fields. With platinum, for example, ions with all charges up to five occur, and with gold all charges up to four. It is of interest that ions of gold and platinum, which have not been found with other methods, are very easily obtained with these sparks.

Ryerson Laboratory,

University of Chicago. March 4.

\section{Interferometer Measurements of the Red Auroral Line 6300}

WE know that considerable interest is attached to certain auroral lines in the region $6300-6400 \mathrm{~A}$. Thus the enhancement of one or more of these lines is responsible for a particular type of red-coloured auror $æ^{1,3}$ and, according to the interpretation of the strong green line by McLennan and his collaborators, the OI-triplet $\left({ }^{1} D_{2}-{ }^{3} P_{0,1,2}\right)$ should appear in this region.

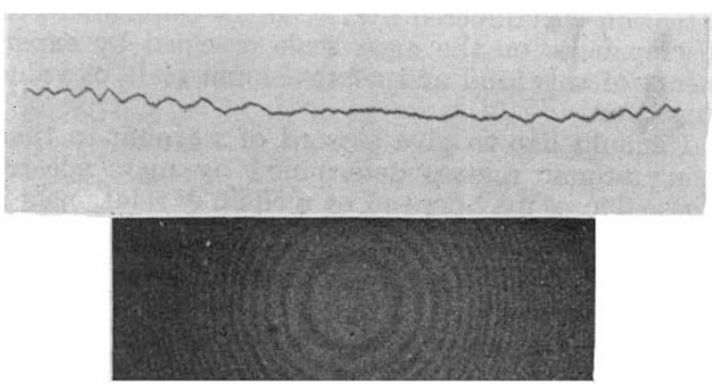

Fra. 1. Interferometer photograph of the red line 6300 and corresponding photometer curve above.

Previous auroral spectrograms taken with instruments of fairly high dispersion have shown two lines, which within the limit of error coincide with the two strongest components of the Or-triplet $t^{3,5}$; and a faint line has been observed which might be identified with the weakest line of the Or-triplet.

The mean of our present measurements gives $\lambda=6300.9( \pm 1)$ for the strongest line $\left({ }^{1} D_{2}-{ }^{3} P_{2}\right)$ and $\lambda=6365.3( \pm 2)$ for the weaker one, which might correspond to $\left({ }^{1} D_{2}-{ }^{3} P_{1}\right)^{3,5}$. Spectrograms in the red ${ }^{4,5}$ indicate that bands belonging to the first positive group appear in the region of the OItriplet.

Some years ago investigations were commenced at the Tromsö Observatory with the object of using interferometer methods for accurate wave-length determinations within the auroral spectrum. Our results relating to the strong green line were dealt with in a recent publication ${ }^{6}$, and we are here going to give a preliminary, brief account of some results which refer to the strongest red line 6300.

Just as in the case of the green line we take advantage of the predominance of this red line, so we can use an interferometer without spectrograph, simply consisting of a Fabry-Pérot quartz plate in front of a camera lens combined with suitable filters and photographic plates. Under these conditions only atomic lines and not the fairly strong bands of the first positive group of $N_{2}$ can give interference fringes.

During an auroral display (January 15, 1934) an interferometer picture of the strong red line was obtained at the Tromsö Observatory with a $2.5 \mathrm{~mm}$. étalon. The microphotometer curve reproduced in Fig. 1 corresponding to a line through the centre shows that the fringe-system is weak, but sufficiently distinct to be measured.

On the same plate were taken two interference pictures of the neon line $\left(\lambda_{\mathrm{vac}}=5854 \cdot 110 \mathrm{~A}\right.$. $)$ in the way and for the purpose described in our previous paper ${ }^{6}$. Comparing the diameters of the rings of the interference picture produced by the aurora with those produced by the Ne-line $5854 \cdot 110$, we may decide that the interference pattern produced by the auroræ must be due to a fairly sharp atomic line situated noar $6300 \mathrm{~A}$, and only the strongest red line $6300 \cdot 9 \mathrm{~A}$. measured on our spectrograms can come into consideration.

The possible error of our spectrographic measurements of the red line, being about $\pm 1 \mathrm{~A}$., interference pictures with one $(2.5 \mathrm{~mm}$.) étaion does not permit us to make an unambiguous determination of the order number $n$ of the rings on the interference pattern. In the interval of uncertainty there are six order numbers which are possible, giving the following values for the wave-length :

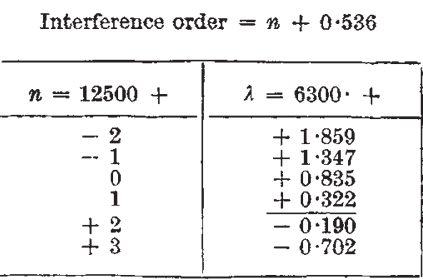

One of our values, $\lambda=6300 \cdot 322$, is nearly equal to that $(6300 \cdot 328)$ derived from the electronic OI states given by Hopfield?. From direct measurements of the red line with a glass spectrograph (dispersion $29 \mathrm{~A} . / \mathrm{mm}$.) Hopfield finds the wave-length $6300 \cdot 23$. As the determination of the electronic levels is based on spectra taken with a dispersion of $1.7 \mathrm{~A} . / \mathrm{mm}$., the wave-length derived from these levels should be most accurate, arid this wave-length is, within the limit of error, equal to one of our values for the wave. length of the red auroral line. 